THE IANCET, J JNGARE $31,18 \% 4$.

塑ecturers

ox

\section{H $\mathrm{O}$ S P I T A L I S M, \\ AND THE CAUSES WHICH DETERMINE THE MORTALITY AFTER AMPUTATIONS.}

Delivered in University College Hospital, Jan. 1874,

BY JOHN ERIC ERICHSEN, F.R.C.S., SENIOR SURGEON TO THE HOSPITAL, AND HOLME PROFESSOR OF CLINICAL SURGERT IN UNIVERSITY COLLEGE, LONDON.

\section{LECTURE III.}

Genthemen,-By overcrowding a condition of the atmosphere will be induced which may, according to the condition of the individuals so congregated together, produce diseases of various types. The overcrowding of uninjured individuals will produce some varieties of typhus. The "gaol fever" of a past generation is an instance of this kind. The overcrowding of wounded people, whether the wound be accidental or surgical matters not, will develop septic disease in one of four forms-namely, hospital gangrene, septicæmia, pyæmia, or erysipelas. That the septic poison which, when once generated, impregnates a wound, and thus gives rise to hospital gangrene, pyæmia, or erysipelas, is capable of transmission through the medium of the atmosphere is undoubted. That it may be generated in the air by overcrowding is equally certain. The results of the most extended and recent observations on the development of this scourge of hospitals in actual warfare, which have of late years been numerous, have left no doubt whatever on the minds of military surgeons that overcrowding is the direct occasion of the whole class of surgical septic diseases. But the word "overcrowding" is liable to misconception, and requires explanation. It is very important to be clear on this point, for by it is not meant the mere heaping together of sick and wounded in one ward or building beyond what it is intended to hold, or only limited by its size; but by "overcrowding," in the sense in which it is here used as the cause of the generation of the septic poison, is meant the accumulation within one ward or under one roof of a greater number of patients than is compatible with such a degree of purity of air as to render it incapable of the development or transmission of the septic poison. It would, in fact, appear that the air of a ward is capable of oxidising and destroying or absorbing a certain amount of morbid emanations from the contained patients; but if these emanations are developed too rapidly or too abundantly the air becomes overcharged with septic matters, and then all the ill-effects of overcrowding at once develop themselves. The contamination of the air of a ward may thus take place even though the actual number of patients lying in it be below what it is constructed to hold, as readily as by the introduction of one single patient beyond the number that the ward is calculated to accommodate with safety. Thus it would be more correct to say that the evil effects of overcrowding, and the special form of disease it will occasion, are rather dependent on the nature than on the actual number of the cases that are contained in any given ward or building at one time. There is no evidence that an accumulation of unwounded patients to any extent can develop hospital gangrene or pyæmia, whatever other disease may thus be generated. Pyæmia could not have been generated in the Black Hole of Calcutta; but a very trifling excess of the number of open suppurating wounds beyond what any given ward is capable of bolding with safety constitutes overcrowding in a sur. gical sense, and will invariably generate septic disease.

I can illustrate this observation from what has happened in this hospital. Ward No. 1. my male Accident Ward, contains a cunic space of 21,924 feet. It is intended to hold fourteen beds, giving a cubic space of 1566 feet to each, or if we deduct the space: occupied by furniture, \&c., about 1500 cubic feet. But not only is the cubic space sufficient, the floor space is also ample. There is about 6 feet between

No. 2631 . each bed, and 10 feet between the opposite rows of lbeds, the ward being 63 feet long by 24 broad. This ward can be well ventilated by a row of windows on each side, a fire-place at one end, and a large door with an open air-hole above, which, however, communicates with the general passages of the hospital at the other end. In this ward we have had many outbreaks of septic disease, pyæmia, and erysipelas, invariably the result of the accidental accumulation in it, not always of too large a number of patients, but of too many severe cases of operation and injury, with wounds in a state of suppuration. The average number of open wounds in this ward is about seven, or half the number of patients it contains, and if these wounds are not severe such a number can be contained in it with moderate safety; but if they rise above this number, or if the majority are severe, then septic disease will certainly break out. The outbreak in Ward No. 1, in November, 1872, which will be fully described hereafter, was produced in this way. The ward contained at the time a remarkably severe set of cases; in fact, the conclusion at which we have arrived is that if this ward contains nine or more cases of open wounds, at least three of which are severe, as amputation or similar cases, an outbreak of septic disease is imminent.

Thus, then, it is the nature of the cases, and not, their number merely, that constitutes the danger of the development of surgical septic disease by so-called overcrowding. Doubtless epidemic influences favour the development of these septic outbreaks that are directly produced by overcrowding. In what these epidemic influences consist $T$ know not, So far as my experience and observation go they are connected with very different meteorological conditions. In wet and in dry weather, in heat and cold, during prevalent easterly and westerly winds, they equally manifest themselves. The existence of these epidemic conditions, in addition to the influence of overcrowding, is evidenced by the admission from out of doors of erysipelatous cases and allied diseases, or by their appearance amongst the outpatients in unusual numbers. But the influence of different meteorological conditions on the development of epidemic septic diseases has jet to be worked out. In reality we know little or nothing that is positive about it. The inflaence of the season of the year is, however, somewhat better ascertained. Thus the observations of Dr. Hewson, of Pbiladelphia, that bave already been referred to, point to the winter months as those in which pyæmia is more rifean observation wbich has been in accordance with my own experience at University College Hospital for a lengthened period, where we have always suffered most from septic disease in the surgical wards during the winter and early spring months. This often appears to be owing, however, less to the direct influence of the season of the year thin to its indirect effect in preventing ventilation, it being difficult in wet or cold weather to get the nurses or patients to submit to have the windows opened so as to allow an influx of fresh air in a constant current, and so prevent the.ward being ventilated wholly from air drawn from the hospital corridors. The air-supply that is thus admitted from the corridors is already in a state of contamination.

Now what is this condition of contamination in hospital air that causes the development of septic disease in a ward, the cubic and floor space of which are sufficient, and which is kept scrupulously clean? That is the next point for inquiry. In the well.known, I may say celebrated, lecture by Prof. Tyndall on " Dust and Disease," that distinguished philosopher points out the important fact that the atmosphere under all circumstances contains floating particles of organic matter; that the motes which dance in the sunbeam are of this character. He states that the air of our London rooms is loaded with this organic dust, nor is the country air free from the pollution; and he goes on to observe that, however much it may disguise itself from ordinary observation, "a powerful beam of electric light causes the air in which the dust is suspended to appear as a semi-solid rather than as a gas. Nobody could in the first instance, without repugnance, place the mouth at the illuminated focus of the electric, beam and inhale the dirt revealed there. Nor is the disgust abolished by the reflection, that although we do not see the nastiness, we are churning it in our lungs every hour and minute of our lives. There is no respite to this contact with dirt; and the wonder is, not that we should from time to time suffer from its presence, but that so small a portion of it would 
appear to be deadly to man." Watts states that a minute quantity of organic matter, one grain in 200,000 , is found in the present mountain air. This increases in the country at a lower level, and in large towns reaches the condition stated by Professor Trudall in the above extract. Even in gea air, according to Dr. Rattray, in the last volume of the Medical-Chirurgical Transactions, it is not absent. This organic matter is the result, in all cases, of vegetable and animal emanations, and it necessarily varies in its composition according to the source from which it is derived. Rattray, in a most interesting paper in the volume referred to, gives an analysis of ship air, and after pointing out a variety of impurities of a gaseous character, states that the more solid impurities are of animal, vegetable, and mineral origin, from the skin, lunge, \&c., of the crew; from the ship, as minute particles of wood, paint, whitewash, leather, \&c. ; from the bilge, containing occasionally minute microscopical animal and vegetable organisms ; from the stores, particles of bread, cotton, wool, \&c., and others which I need not mention. He states that it is the volatile organic matters thrown off by the skin which give the ship air its well-known and of ten nauseous smell. Dr. Parkes, in his admirable work on Practical Hygiene, gives the following account of hospital air. At page 88, he says: " 1 have examined the air of various barracks and military hospitals, and have detected large quantities of epithelium from the skin, and, perhaps, the mouth." At page 99 , he says: "The most important class of diseases produced by impurities in the atmosphere is certainly caused by the presence of organic matters floating in the air, since under this heading come all the specific diseases. The exact condition of this organic matter is unknown; whether it is in the form of impalpable particles, or of moist or dry epithelium and pus-cells, is a point for further inquiry." And again, at page 106, he says : "The air of a sick ward, containing as it does an immense quantity of organic matter, is well known to be most injurious. At a certain point of impurity erysipelas and hospital gangrene appear. The occurrence of either disease is, in fact, a condemnation of the sanitary condition of the ward." Again, at page 100, he states that " erysipelas and hospital gangrene in surgical wards are often carried by dirty sponges, dressings, \&c. Another mode of transference is by the passage into the atmosphere of disintegrated pus-cells and putref ying organic particles, and hence the great effect of free ventilation in military ophthalmia, in erysipelas, and bospital gangrene." Lastly Dr. Douglas Cunningham, of Calcutta, has recently found that the atmospheric dust largely consists of spores of fungi, and that the majority of these are living and capable of growth and development; and that bacterial matter exists also in dry dust, so that when this is added to putrescible fluids a rapid development of fungi and bacteria occurs. Here then we find, on the combined authority of able and recent observers, abundant evidence not only of the existence in the atmosphere of large quantities of suspended organic matters, but of animal débris and exfoliations, and of other animal particles, capable under favourable circumstances of generating septic diseases.

We are still in complete ignorance as to the precise nature of the septic poison that produces those various forms of disease that we recognise as being due to its influence. We know it, in fact, by its effects, but are ignorant of what it essentially consists. That there are different forms of septic disease appears more than probable. We find that the different varieties of septic diseases are not interchangeable, but are as distinct in themselves, in the symptoms they present, in the course they run, in the pathological conditions that are found, as any of the other zymotic diseases. In whatever way originating and in whatever it may essentially consist, there can be little doubt that this septic virus is communicable from patient to patient through the medium of the organic particles of various kinds with which the atmosphere of any crowded building, be it hospital, or barrack, or man-of-war, is in. variably charged. And when we reflect on the exceedingly minute, infinitesimal, in fact inappreciable, quantity of any animal virus, as that of cow-pox, small-pox, or syphilis, that is needed to communicate its own disease when applied to a fitting soil, we can easily understand how the virus of the septic diseases that occur in surgical wards may be transmitted from wound to wound, even on so slight a vehicle as atmospheric dust.
There are four of these septic diseases universally re. cognised by surgeons-viz., hospital gangrene, septicæmia, pyæmia, and erysipelas. Let us very briefly study the mode of development of them.

With regard to hospital gangrene I need say but little. It is universally recognised by the concurrent testimony of military surgeons that this disease originates in the first instance as a direct consequence of overcrowding of the wounded in hospitals that are insufficiently ventilated. The experience of the Franco-German war added confirmation, if any were needed, to this view of the origin of the pestilence, which, however, when once generated, is capable of indefinite propagation by contact through the medium of the fingers, instruments, sponges, and surgical appliances. The occurrence of hospital gangrene in civil hospitals is now, fortunately, extremely rare, and its development in such institutions is of itself an evidence that the sanitary condition of the building is, for the time at least, in a bad state. The repeated occurrence of hospital gangrene in a civil hospital would undoubtedly be discreditable to those who had the management of its sanitary arrangements. In University College Hospital we have now had no outbreak of hospital gangrene for more than twenty years, and I trust never again to see it here as having been developed within the walls of the hospital. It is altogether a preventable disease, and ought never to occur in any institution that is conducted on sanitary principles. The few cases that we have had of late years have been brought into the hospital from without, and, curiously enough, last summer two cases came to us, of a mild form certainly, from a convalescent institution.

Septicæmia is not of very common occurrence, but yet there is a certain percentage of deaths occurring from it. In this hospital we have abnut one death in the year from this cause. Its influence on the general mortality after operations is therefore but trifling, but its existence is an indication of a septic influence, and I believe that a certain number of cases of low, irritative fever following operations and injuries partake of a septicemic type. In fact, the gradation from ordinary traumatic fever into septicæmia is easy, and I believe not very unfrequent. Surgeons themselves suffer at times from small whiffs of this poison. Who that has been long connected with a hospital has not at times, after the examination of a sloughing cancer, or some other horribly putrescent and foul disease, felt feverish, depressed, prostrated for some hours, or a day or two, conscious of the absorption of a poisonous effluvium, which after a period of febrile depression eliminates itself from the system by an attack of offensive diarrhoea, the eruption of a pustule, or perhaps by some more distinctly localised inflammatory action, as tonsillitis, or a boil? The cause of septicæmia appears, however, to me to be somewhat obscure. It does not seem to be distinctly connected with overcrowding, but rather with the development of putrescent discharges from unhealthy or malignant ulcers. The offensive discharge from ulcerated cancer uteri is supposed by some to tend very specially to its production, and it has been a cause of death af ter ovariotomy when the operation has been practised in the same building in which a woman suffering from that disease is lying.

But the two most important of the septic diseases are undoubtedly pyæmia and erysipelas. These two diseases are commonly looked upon as being more or less allied, and they may be so in so far as their etiology and those epidemic influences that predispose to their occurrence are concerned, but in all other respects they differ widely.

Acute pyæmia is essentially a hospital disease. As has already been stated in the last lecture, it seldom occurs except in hospital practice; and perhaps the best proof that we have of the rarity of the occurrence of acute pyæmia out of hospital exists in the rarity of the admission of a pyæmic patient into a hospital from without whilst labouring under the disease. Almost every case of pyæmia that $I$ have seen in hospital practice has originated within the building itself By reference to Table $B$ it will be seen that the amount of pyæmia varies very greatily in different London hospitals, which is an additional proof of its being dependent for its origin on conditions that are more rife in some institutions than in others. So far as University College Hospital is concerned, there appears to be at present a tolerably uniform amount of it. In three years, from July, 1870, to July. 1873, we had twenty-three cases: nine in connexion with 
amputations, fourteen with other operations and injuries. Of these twenty-three cases, four occurred in the latter half of 1870 , eight in 1871 , eight in 1872, and three in the first half of 1873. As to the mode of its development, there can be no doubt that it is the result of the exposure of wounds to an atmosphere overcharged with organic matter emanating from other patients with suppurating wounds. What I stated in the last lecture about the infection of the military hospitals with pyæmia during the Franco-German war, and the comparative freedom of the hut hospitals from this disease, points clearly to the cause. I look upon pyæmia, when of traumatic origin, as being primarily the local septic impregnation of a wound by atmospheric matter in a condition capable of developing changes of such a character in the wound that its fluids decompose, its surface becomes foul and sloughing, and the veins leading from it become plugged with soft clots, putrid and easily decomposable. The constitutional symptoms of this dread disease-the prostrating rigors, the profuse transudations from skin and lung saturating the bedclothes and contaminating the air around with a faint, sickly odour, the high temperature, the extreme mental depression-are all consequent on the entry into the circulation of the septic virus deposited from air upon wounds, thence absorbed into the veins, and then transmitted through the system.

Pyæmia stands next to hospital gangrene amongst septic diseases of local origin. It is less marked in its local phenomena; it is far more developed in its constitutional symptoms, but though less marked locally it is most distinctly characterised. Mr. Beck, who has made the postmortem examinations of the twenty-one fatal cases of pyæmia that have occurred in University College Hospital during the past three years with a degree of minuteness and care that leave nothing unnoted, states that in every case except one-a case of necrosis of the tibia, in which there was no open wound until a few hours before deathdistinct local evidences were found. In fourteen cases the local origin of the disease was evidenced by brokendown and putrid clots in the veins leading from the part, and in six there were foul sloughing wounds. It is a remarkable fact that acute pyæmia never appears to occur in single isolated cases, but invariably in groups of two or three, not necessarily absolutely contemporaneous, but only separated by short intervals of time.

Is pyæmia contagious? The French surgeons generally believe that it is highly so. I have never seen an unequivocal instance of it. This, however, may be accounted for by the fact that whenever a case of pyæmia has developed itself in this hospital immediate steps have been taken to guard against contagion by isolating the patient and disinfecting the ward. We act, in fact, as if its contagion were proved, although we may not in reality be in possession of this proof. There certainly appears to be an epidemic influence that favours its development. It is of ten coincident, though not invariably so, with outbreaks of erysipelas both in the hospital itself and out of doors. I have already said that it is necessary for the surgeon to consider the nature rather than the number of the cases contained within a surgical ward. This is well exemplified by an outbreak of pyæmia that took place in Ward 1 in January, 1871. In the early part of the month this ward contained a rather large number of severe wounds, including a sloughing cancer in the groin, an amputation of the leg, a compound fracture of the fibula with extensive laceration of the soft parts, a compound fracture of the femur, and one of the tibia. Pyæmia occurred in the case of amputation of the leg on the 19th of January, in the compound fracture of the fibula on the $3 \mathrm{rd}$ of February, in the compound fracture of the femur on the 13th, and all these patients died. At notime, however, was the ward overcrowded as to number of patients, two out of fourteen beds being empty the whole time, so that each patient had a cubic space of no less than 1.800 feet of air. But the ward contained for two months an average of seven or eight open wounds, of which five were always severe, such as amputations or compound fractures. During this period there was only one case of erysipelas, and the hospital generally was free from that disease. Another instance of a similar kind occurred in December, 1872. At this period Ward 1 became again crowded with severe wounds, there being an average of nine open wounds, seven or eight of which were severe, including three amputations and an extensive necrosis of the tibia. One of the amputation cases had pyæmia and recovered, the only instance of recovery from pyæmia during the last three years and a half. Another had pyæmia and died. On this occasion erysipelas broke out in three cases. In both these outbreaks the weather was cold.

Now in erysipelas we find various influences tend to develop the disease. Contagion, overcrowding, and epidemic influences all produce their effect. About the contagion of erysipelas there can be no question. I could adduce many instances of it, but one especially occurs to me. Though it happened many years ago, it made.a deep and lasting impression upon my mind. I will read an extract from the notes I took at the time. On the 17th of January, 1851, a case of phlegmonous erysipelas of the leg was accidentally brought into Ward $1 . \mathrm{As}$ soon as the nature of the disease was discovered the patient was removed to the exysipelas ward at the top of the building, having only remained in Ward 1 for about two hours. At this time Ward 1 was perfectly healthy, but in a few days after, a patient lying in the next bed to that into which the erysipelatous patient had been taken, and who had been operated on for necrosis of the ilium, was seized with erysipelas. On the 22 nd I performed five operations on patients who were in this ward. - Of these cases three were attacked by erysipelas on the 24th-viz, a case of necrosis of the tibia, one of partial amputation of the foot, and one of encysted tumour of the scalp. All these patients died. On the 24,th. a patienl was operated on by me for strangulated femoral hernia in this ward. He was seized with symptoms of low peritonitis, doubtless of an erysipelatous character, and died on the 31st. No case of pyæmia showed itself, but all the patients in the ward who were not attacked by erysipelas had diarrhœa and severe gastro-intestinal irritation. Here were a series of lamentable catastrophes doubtless directly introduced by the accidental intrusion of an erysipelatous patient into an operation ward, the disease being then propagated by contagion, though he only remained for two hours in the ward. That erysipelas, however, may be developed in other ways there can be little question. I believe that by no means an uncommon cause of infection occurs from dissecting students. I have several times seen cases, both in hospital and private practice, distinctly referable to contamination from this cause, and I think that no student, during the time that he is engaged in dissections, should be allowed to act as a dresser in a hospital. There is a remarkable resemblance, if not an actual similarity, between puerperal peritonitis and erysipelatous inflammation of the peritoneum following operations on the organs contained in that cavity. The influence of contamination in the way I mention is well illustrated by what occurred some years ago at the Vienna Hospital, and is described by Dr. Braidwood in his work on Pyæmia. Prior to 1846 the number of deaths amongst lying-in women in this hospital was 1 in 10 . In order to check this frightful mortality, Dr. Senelweiss required the students who attended these women to avoid making autopsies, and obliged them to wash their hands in a solution of chlorine before and after making vaginal examinations. The result was that the mortality in 1848 had fallen to 1 in 74 .

That erysipelas is often of epidemic origin there can be no question, but the influence of any epidemic is immensely increased by unhealthy conditions of a ward from overcrowding. Of this I will give you one illustration. In the middle of November, 1872, ward No. 1 was filled by a very severe set of cases. There was one patient who had had both legs amputated below the knees; another whose leg bad been amputated through the knee; another who had a compound fracture into the ankle-joint; one with extensive necrosis of the tibia; and another with extensive laceration of the forearm. And this condition of things was kept up during three primary operations-one of the foot and the others of the thigh; these cases being admitted in December, but whilst the preceding ones were under treatment. There was no erysipelas in the ward until Nov. 15th. On that day the patient with compound fracture into the ankle was attacked. He recovered, and the erysipelas had left him by the 21 st. On this day the patient with necrosis of the tibia had the sequestrum removed: he was a feeble old man; he was attacked by erysipelas on the 1st, was removed to the erysipelas ward, and died on the 7th of December. On the 4 th of December the patient with the 
scalp wound was attacked, and was at once removed to the erysipelatous ward. During this period-namely, Dec. 7th and the 22nd-two of the other patients, the one with an amputation through the knee-joint and another, were seized with pyæmia. Only one out of the twenty-three patients with acute traumatic pyæmia recovered. During the same period two of my patients in Ward 5, both suffering from chronic wounds, one of fistula and another with a sinus in the thigh, were attacked by erysipelas. To them it is possible that the disease was convejed by the house-surgeon or dressers. But as other cases of erysipelas occurred in the hospital at the same time, and one was admitted from out of doors, it is probable that an epidemic influence existed.

In the next lecture I shall proceed to the consideration of the circumstances by which the fatality may possibly be lessened, and of the influence of treatment in diminishing septic disease in hospitals.

Note.-In the last lecture it should have been stated that primary amputation of the thigh at the.hip-joint is not admissible in adults; in children it has succeeded.-J.E.E.

\section{All didders}

\section{PY EMIA IN PRIVATE PRACTICE.}

Delivered before the Clinical Society of London, January 23rd, 1874,

BY PRESCOTT HEWETT, F.R.C.S., PRESIDENT OF THE SOCIETY, SENIOR SURGEON TO ST. GEORGE'S HOSPITAL, ETO.

Gentumyer,-In taking the chair this evening, I cannot refrain from thanking you for the honour which you have again conferred upon me in placing me in this position. I confess I had my misgivings as to my fitness when $I$ took the chair last year; but whatever may have been my shortcomings, thanks to the Council, and more especially to the unwearied zeal of our secretaries and our treasurer, I am happy in being able to congratulate you upon the still increasing prosperity of the Clinical Society.

With these few but heartfelt words, I pass on to the more immediate business of this evening, and in doing so I shall follow out the course which I ventured to enter upon last year, and will again give you some gleanings from private practice the clinical results of which may be made valuable, and all the more so when contrasted with those of hospital practice.

The subject to which I shall direct your attention is that of Pyæmia, a subject than which none is more important, and the consideration of which, in some of its bearings, has of late years been largely occupying the attention of our profession. The few observations which I have now to offer you on this subject will be confined altogether to cases occurring in private practice.

A young lady, aged fifteen, stoutish, but of good general health, came under my care for congenital cystic tumour at the root of her neck. When an infant an attempt had been made to remove this tumour, but a bit of it was left, as it was closely adherent to the large vessels. For several years after this operation nothing was noticed in relation to this tumour; but for two or three years previous to my seeing the patient it had, without any apparent cause, taken to growing; and when I was consulted it occupied the whole of the lower part of the left side of the neck, projecting beyond the elavicle. At a consultation with Mr. Keate, who performed the operation, it was determined that singlethread setons should now be used, and two of these were introduced into the tumour. In a few days intense inflammation set in, and suppuration followed. A quantity of matter was let out; the swelling subsided, and for some days everything appeared to be going on satisfactorily: then came rigors and sweating, but without any increase in the local trouble. After a while it became evident that the patient was suffering from pyæmia, the mischief being in the left lung; and for several days her condition was most perilous. But one afternoon, after a violent fit of coughing, a large quantity of offensive matter was suddenly brought up, after which the more dangerous symptoms gradually subsided, and the patient ultimately recovered. She remained perfectly well, without any further trouble as to the tumour. In this case the patient's room was large, lofty, and well ventilated, and was in a well-found house in one of the best streets in town.

A hale old gentleman, close upon eighty, who had long enjoyed excellent health, had a small warty growth on the skin just over the insertion of the tendo Achillis. This growth I removed, as it constantly became chafed and troubled him much in walking. I made him lay up for a few days. As the trifling wound was all but healed, he was allowed to go about a little; but being of very active habits, he one day took a longer walk than usual. This was followed by some slight inflammation of the wound, which, however, soon subsided. He was on the eve of returning to his usual avocations, when rigors and sweatings made their appearance. Then came an immense deep-seated abscess in the thigh, under the constant drain of which he ultimately sank.

I was called in consultation to a gentleman who was suffering from pyæmia, who a short time before had had a small wart removed from the scrotum. He died. The details of the case are given further on, as I had attended this gentleman three or four years previously for a former attack of pyæmia.

Shortly after I began practice I removed a small sebaceous tumour from the scalp of a lady. Everything went on well for the first few days, then came erysipelas of the scalp, subsequently followed by pyæmia and death. As far as one could judge, the conditions under which this trivial operation was performed were all favourable. The patient was middle-aged and not stout. She had some months previously undergone, without a bad symptom, a similar operation for the removal of three other sebaceous tumours. The bedroom was in a house in one of the most open and healthiest of our squares, and was large and well ventilated. The weather was temperate, and the patient had remained in the house after the operation.

I operated upon a middle-aged lady of good general health for scirrhus of the breast, and for the first eight days everything looked promising; then came rigors, followed by erysipelas around the wound, which for a few days went on slowly spreading. Subsequently pyæmia came on, and under this she gradually sank. The house in which the operation was performed was in one of the best streets in Pimlico; the bedroom was of a good size, and well ventilated.

About a month afterwards I operated upon another lady for scirrhus of the breast. She was middle-aged and slim. She was of a sallow complexion, and of good general health. In this case, too, everything promised well for the first few days. Then came severe rigors and sickness. Erysipelas soon made its appearance around the wound, from whence it gradually spread. Matters went on thus for a few days then there was pyæmia, and in a few days more the patient was dead. In this case the operation was performed a few miles out of town. The bedroom was large and well ventilated, in a large and well-built house, with all modern appliances, and situated on a heath on an elevated ground, overlooking a wide expanse of country. As far as one could judge, no better or healthier situation could have been selected for an operation.

A lady, aged about forty-five, had a large sero-cystic tumour of the breast, some cysts of which occasionally suppurated, and under such circumstances were sometimes opened and sometimes allowed to burst. Matters went on thus, as she would not hear of an operation, for between eight and nine years, her general health being as good as ever between the attacks. At this period a small cyst suppurated and was allowed to burst; shortly afterwards erysipelas made its appearance round the edge of the little sore. Then in a few days came rigors and sweating, with pain and great $\mathrm{swelling}$ in one of the knee-joints. In a few days more this lady sank. At the time of the bursting of the cyst the patient was in her usual good health. Her bedroom was of fair size and well ventilated, in a bouse in a part of town generally considered to be one of the healthiest.

A young gentleman met with an accident to his shoulder which led to inflammation of the joint, and for this he ulti- 